Rapid Communications

\title{
3,3',5,5'-Tetramethylbenzidine Oxidation on Paper Devices for Horseradish Peroxidase-based Assays
}

\author{
Lori Shayne Alamo Busa, ${ }^{* 1, * 2}$ Takeshi Komatsu, ${ }^{* 1}$ Saeed Mohammadi, ${ }^{* 1}$ Masatoshi Maeki,*3 \\ Akihiko ISHIDA, ${ }^{* 3}$ Hirofumi TANI, ${ }^{* 3}$ and Manabu ToKESHI $* 3, * 4, * 5, * 6 \dagger$ \\ *1 Graduate School of Chemical Sciences and Engineering, Hokkaido University, Kita 13 Nishi 8, Kita, \\ Sapporo 060-8628, Japan \\ *2 Physical Sciences Department, College of Arts and Sciences, Nueva Vizcaya State University, Bayombong, \\ Nueva Vizcaya 3700, Philippines \\ *3 Division of Applied Chemistry, Faculty of Engineering, Hokkaido University, Kita 13 Nishi 8, Kita, \\ Sapporo 060-8628, Japan \\ *4 ImPACT Research Center for Advanced Nanobiodevices, Nagoya University, Furo-cho, Chikusa, \\ Nagoya 464-8603, Japan \\ *5 Innovative Research Center for Preventive Medical Engineering, Nagoya University, Furo-cho, Chikusa, \\ Nagoya 464-8601, Japan \\ *6 Institute of Innovative for Future Society, Nagoya University, Furo-cho, Chikusa, Nagoya 464-8601, Japan
}

\begin{abstract}
We report on the colorimetric oxidation of 3,3'5,5'-tetramethylbenzidine (TMB) by hydrogen peroxide using horseradish peroxidase on photolithography-fabricated (P-PAD) and wax-printed (W-PAD) paper-based analytical devices. Fabricating PADs via photolithography exposes the hydrophilic areas to polymers (photoresists) and solvents, not only reducing the hydrophilicity, but also affecting the TMB- $\mathrm{H}_{2} \mathrm{O}_{2}$ assay system with an unavoidable incomplete elimination of photoresist during fabrication. Detection signals are then observed in the presence of photoresist residues on the P-PAD, even at a blank HRP concentration.
\end{abstract}

Keywords TMB oxidation, horseradish peroxidase, paper-based analytical devices, photolithography, wax-printing

(Received July 14, 2016; Accepted July 15, 2016; Published August 10, 2016)

\begin{abstract}
Microfluidic paper-based analytical devices ( $\mu$ PADs) have gained much attention in clinical and analytical fields of research due to the many advantages that they provide, including rapid detection with the use of low-cost, portable and easy-to-use devices that make them highly applicable for on-site testing, more specifically in resource-limited settings. ${ }^{1-5}$ A number of $\mu P A D$ fabrication methods have been developed to define hydrophilic microchannels on $\mu$ PADs, including photolithography, ${ }^{6,7}$ plasma-etching, ${ }^{8}$ wax-printing, ${ }^{9-11}$ plotting, ${ }^{12}$ screen-printing, ${ }^{13,14}$ contact stamping, ${ }^{15-17}$ cutting, ${ }^{18-20}$ and laminating, ${ }^{21,22}$ among others. Each fabrication method has its own advantages and limitations. Photolithography was the first reported fabrication method for $\mu$ PADs with the main advantage of producing clearly defined hydrophilic channels of up to $186 \pm 13 \mu \mathrm{m}$ width and hydrophobic barriers formed after polymerization of the photoresist. ${ }^{6}$ This method, however, has disadvantage of exposing the hydrophilic areas to polymers and solvents during the fabrication process, ${ }^{23}$ not to mention requiring a longer fabrication time and expensive instrumentation (Table S1, Supporting Information). As the development of $\mu$ PADs progressed, simpler fabrication methods have been reported, such as wax-printing, which provides rapid $(\sim 5 \mathrm{~min})$
\end{abstract}

$\dagger$ To whom correspondence should be addressed.

E-mail: tokeshi@eng.hokudai.ac.jp fabrication and simply requires a wax printer and hotplate, or oven to melt the wax on the paper. With the latter fabrication method, the exposure of hydrophilic areas to polymers and solvents is avoided, which minimizes any probable disadvantages that the fabrication procedure might bring to the $\mu$ PADs during an assay. This includes alteration of the wicking properties of the paper as to those fabricated via photolithography.

In the present work, we fabricated paper-based spot arrays on Whatman ${ }^{\mathrm{TM}}$ chromatography paper grade 1 via photolithography and wax-printing methods. A horseradish peroxidase assay similar to our previous report based on the catalytic oxidation of 3,3',5,5'-tetramethylbenzidine (TMB) by hydrogen peroxide is described using the fabricated devices. ${ }^{23}$ Here, we focus on the effect of the photoresist and solvent exposure of the hydrophilic areas of the photolithography-fabricated paper-based analytical devices (P-PADs) to detection signals produced as compared to the un-exposed hydrophilic areas that were fabricated via waxprinting (W-PADs). The results are discussed.

All reagents were of analytical grade. TMB chromogen reagent $(35.7 \mathrm{mM}$; Dojindo Laboratories) was prepared with acetonitrile. The $1 \times$ blocker bovine serum albumin in phosphatebuffered saline (BSA-PBS) (Thermo Fisher Scientific Inc.) was diluted with $1 \times$ phosphate-buffered saline Tween ${ }^{\circledR} 20$ (PBST) (Thermo Fisher Scientific Inc.), $\mathrm{pH} 6.5$ (the $\mathrm{pH}$ was adjusted using $0.2 \mathrm{M} \mathrm{HCl}$ ), which was also used as the washing solution 


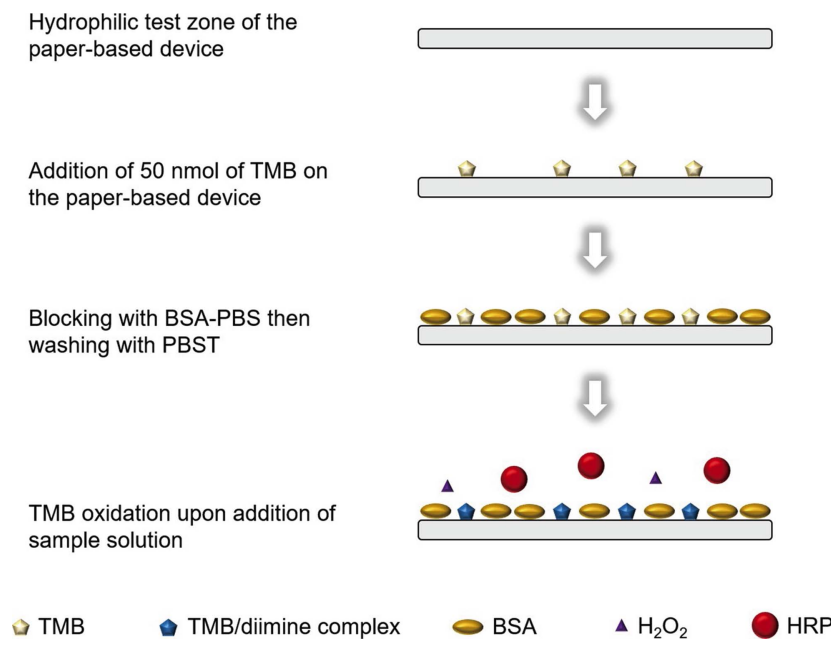

Fig. 1 Schematic illustration of a colorimetric assay procedure involving the catalytic oxidation of TMB by hydrogen peroxide on PADs.

during the assay. Also, $1 \mathrm{mg} \mathrm{mL}^{-1}$ of a HRP stock solution (Wako Pure Chemical Industries, Ltd.) was prepared by dissolving the flakes in PBST. Increasing concentrations of HRP standards were prepared by diluting the stock solution with PBST containing $0.001 \%$ hydrogen peroxide.

Paper-based analytical devices (PADs) were fabricated via photolithography, as described in our previous work, ${ }^{23}$ with a slight modification of the first reported method, ${ }^{1}$ and via waxprinting, as described previously, ${ }^{10}$ with a slight modification. In brief, for the P-PAD, the Whatman ${ }^{\mathrm{TM}}$ chromatography paper (GE Healthcare Life Sciences) was first immersed in SU-8 2010 photoresist (Microchem) for about $30 \mathrm{~s}$, spun for $5 \mathrm{~s}$ at $500 \mathrm{rpm}$, then for another $30 \mathrm{~s}$ at $2000 \mathrm{rpm}$ using a spin-coater (Mikasa MS-A100), prebaked at $95^{\circ} \mathrm{C}$ for $5 \mathrm{~min}$, aligned under a mask and exposed to UV light for $18 \mathrm{~s}$, post baked at $95^{\circ} \mathrm{C}$ for another 5 min, developed using the SU-8 developer (Microchem) for $5 \mathrm{~min}$, washed three times with isopropanol, and finally dried using high-pressure air. The patterns of the photomasks were designed using an AutoCAD 2015 (Autodesk, Inc.), and then ordered from Unno Giken Co., Ltd. for printing with resolutions of $12700 \mathrm{dpi}$ each. For the W-PADs, the patterns were first designed using Inkscape software, printed on the chromatography paper using a wax-printer (Tektronix Phaser 850), and then the wax was melted into the paper at $100^{\circ} \mathrm{C}$ for 2 min using an oven (Yamato Drying-oven DX-38) to form hydrophobic barriers.

Using both the P-PAD and W-PAD, we simultaneously performed a simple HRP assay via a spot test method. Figure 1 shows a schematic illustration of the assay procedure. Firstly, $1.4 \mu \mathrm{L}$ of a $35.7 \mathrm{mM}$ TMB solution (equivalent to $50 \mathrm{nmol}$ of TMB) was added onto each test zones of the paper-based arrays. After at least 2 min of drying, $10 \mu \mathrm{L}$ of a blocker, BSA-PBS, was next added onto each test zone. After 20 min of incubation, the test zones were washed three times with $7.5 \mu \mathrm{L}$ each of PBST. After $15 \mathrm{~min}$ of air drying at ambient temperature, $5 \mu \mathrm{L}$ of the HRP standard solutions $\left(0-1000 \mathrm{ng} \mathrm{mL}^{-1}\right)$ containing $0.001 \%(\mathrm{v} / \mathrm{v})$ hydrogen peroxide in PBST were added into the respective test zones. The images of the PADs were recorded using a digital camera (EOS Kiss X6i Canon) and analyzed using ImageJ software (NIH), as described previously. ${ }^{23}$

The catalytic oxidation of TMB by hydrogen peroxide on PADs produces blue-colored products that can be easily detected by the naked eye. ${ }^{23}$ As described previously by Josephy et al.,${ }^{24}$ the blue-color production is caused by the one-electron oxidation
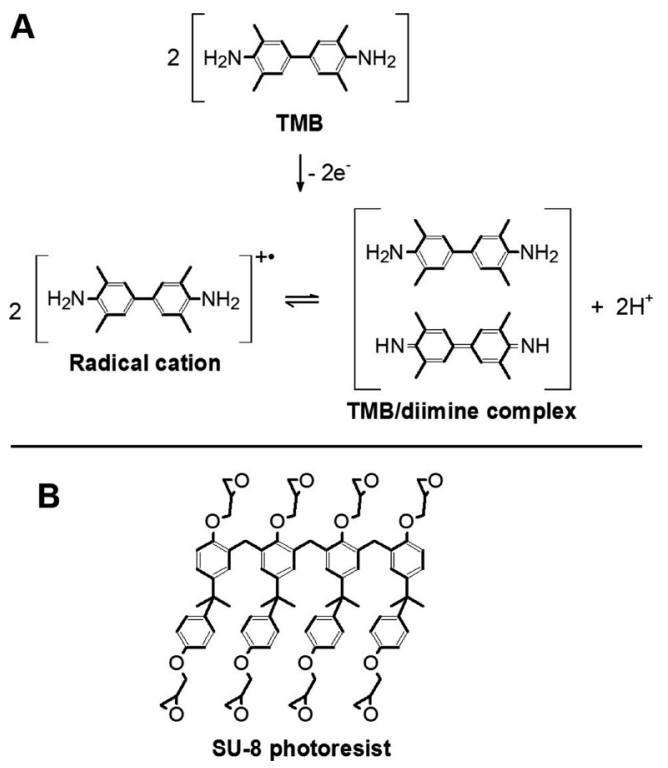

Fig. 2 (A) Catalytic oxidation of TMB by hydrogen peroxide producing the blue-colored TMB/diimine complex. ${ }^{24}$ (B) Chemical structure of SU-8 photoresist.

of TMB in solution, producing a blue charge-transfer complex of the parent compound and its imine oxidation product in equilibrium with its cation free-radical in the presence of less than equimolar hydrogen peroxide (see Fig. 2A for structures). However, the PADs that are fabricated via photolithography have hydrophilic areas that are exposed to polymers and solvents, specifically to the SU-8 photoresist, a strong electrondonor consisting of 8 epoxy groups (Fig. 2B), which causes the production of detection signals from the possible formation of a charge-transfer complex with TMB. As observed experimentally, even prior to the addition of a sample solution consisting of HRP and hydrogen peroxide, the simple addition of TMB on the hydrophilic test zones of the PADs produce a slightly blue color intensity. This observation may be attributed to the unavoidable incomplete elimination of the photoresist during fabrication.

We further confirmed such an observation by comparing the experimental results using PADs that were fabricated via photolithography, in parallel with PADs that were fabricated via wax-printing. As described previously, photolithography has the disadvantage of exposing the hydrophilic areas of the PADs to polymers and solvents, which greatly reduces the hydrophilicity and wicking ability of the PADs after fabrication. ${ }^{23}$ Therefore, although this work presents a spot test method of detection, the need for blocking was necessary to increase the hydrophilicity of the chromatography paper after being subjected to photolithography. The blocking also enables the $5-\mu \mathrm{L}$ volume of the sample solution to spread throughout the 5-mm diameter test zones. As the HRP assay was further performed on both the P-PAD and W-PAD, we have observed notable differences on the signal intensities produced at a similar HRP concentration range. Figure 3 shows a comparison of the detection signals observed using the two PADs. At very low HRP concentrations, including the blank, the P-PAD produced light-blue detection signals. Analyzing the images at the blank concentration using ImageJ software shows a higher cyan intensity using the P-PAD (Fig. 3B) compared to that using the W-PAD (Fig. 3C). This may be attributed to the photoresist residues on the P-PAD, which possibly formed a charge-transfer complex with TMB, therefore producing the faint-blue false detection signal. 


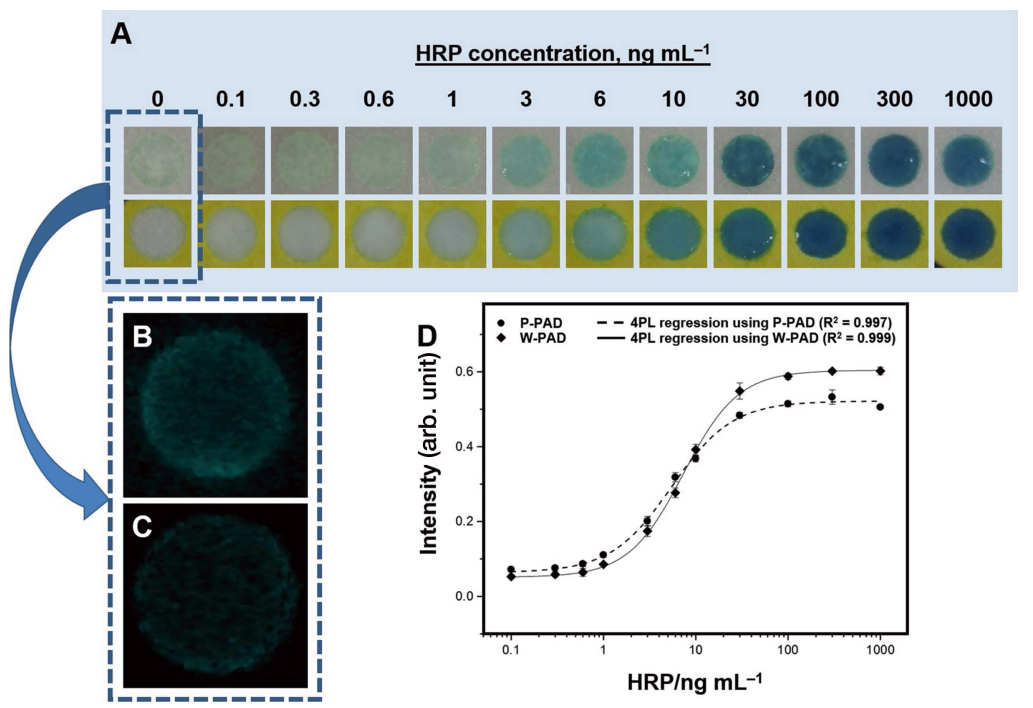

Fig. 3 (A) Representative images of spot tests performed on P-PAD (top images) and W-PAD (bottom images) at increasing HRP concentration. (B) and (C) Respective cyan profiles of images of the fabricated devices at blank HRP concentration. The cyan profiles were enhanced by adjusting the contrast and brightness to $-20 \%$ and $+40 \%$, respectively. (D) 4-Parameter logistics nonlinear regression curve for HRP determination. The error bars represent 4 replicate measurements.

Another possibility could be that the amine groups of TMB may have interacted with the epoxy groups of the photoresist, and thus facilitating early deprotonation of the amine groups upon the addition of TMB to the P-PAD (Fig. 2). Then, the early formation of the deprotonated TMB could have formed a charge-transfer complex with the free TMB, resulting in the formation of a higher background signal (i.e., detection signal at blank concentration). However, as the HRP concentration increased, the detection signals also increased, but the magnitude of the intensities were reversed, showing higher intensities with the polymer-free test zones of the W-PAD from 10-1000 ng $\mathrm{mL}^{-1}$ HRP concentration than with those of P-PAD. This may be due to a decrease in the available TMB concentration for the reaction to proceed at the hydrophilic test zone of the P-PAD, owing it to possible binding of the TMB molecules to the photoresist, and hence, the production of lower signal intensity. Yet, computing for the detection limits (LODs) of HRP (blank intensity plus 3-times the standard deviation) on both PADs via 4-parameter logistic (4PL) nonlinear regression using OriginPro software (OriginLab Corporation) show a lower value for the P-PAD $\left(0.33 \mathrm{ng} \mathrm{mL}^{-1}\right.$ or $\left.0.038 \mathrm{fmol}\right)$ compared to that of W-PAD (1.08 $\mathrm{ng} \mathrm{mL}^{-1}$ or $\left.0.12 \mathrm{fmol}\right)$. The lower LOD for the P-PAD may be attributed to the sigmoidal shape of the 4PL calibration curve with the determined inflection point of $5.47 \mathrm{ng} \mathrm{mL}^{-1} \mathrm{HRP}$ and Hill slope (referring to the steepness of the curve) of $1.32 \mathrm{ng}^{-1} \mathrm{~mL}$, which are lower values compared to those of W-PAD (7.33 $\mathrm{ng} \mathrm{mL}^{-1}$ HRP and $1.46 \mathrm{ng}^{-1} \mathrm{~mL}$, respectively). Nevertheless, visual analysis reveal that colorimetric detection for $\mathrm{HRP}$ using the TMB- $\mathrm{H}_{2} \mathrm{O}_{2}$ assay system on W-PAD presents more reliable readouts using the naked eye than on P-PAD.

The colorimetric oxidation of 3,3',5,5'-tetramethylbenzidine by hydrogen peroxide in the presence of horseradish peroxidase using photolithography-fabricated and wax-printed paper-based analytical devices is reported. The production of a faint detection signal, even at blank intensity, suggests the possible formation of a blue colored complex of TMB and the photoresist residues on P-PAD. However, at higher HRP concentrations, more intense detection signals are produced using W-PAD at concentrations from 10 to $1000 \mathrm{ng} \mathrm{mL}^{-1}$, which may be attributed to possible binding of the TMB molecules to the photoresist residues on the P-PAD, and hence, reducing the available TMB concentration for the reaction, and producing less intense detection signals. These findings therefore demonstrate that the use of W-PADs for the TMB- $\mathrm{H}_{2} \mathrm{O}_{2}$ assay system in the presence of HRP offers more reliable visual readouts for practical applications involving HRP-labeled molecules in clinical and bioanalytical fields of research.

\section{Acknowledgements}

This research was supported by the Urakami Foundation for Food and Food Culture Promotion.

\section{Supporting Information}

This material is available free of charge on the Web at http:// www.jsac.or.jp/analsci/.

\section{References}

1. A. W. Martinez, S. T. Phillips, M. J. Butte, and G. M. Whitesides, Angew. Chem. Int. Ed., 2007, 46, 1318.

2. S. Mohammadi, M. Maeki, R. M. Mohamadi, A. Ishida, H. Tani, and M. Tokeshi, Analyst, 2015, 140, 6493.

3. D. M. Cate, J. A. Adkins, J. Mettakoonpitak, and C. S. Henry, Anal. Chem., 2015, 87, 19.

4. A. K. Yetisen, M. S. Akram, and C. R. Lowe, Lab Chip, 2013, 13, 2210.

5. L. S. A. Busa, S. Mohammadi, M. Maeki, A. Ishida, H. Tani, and M. Tokeshi, Micromachines, 2016, 7, 86.

6. A. W. Martinez, S. T. Phillips, B. J. Wiley, M. Gupta, and G. M. Whitesides, Lab Chip, 2008, 8, 2146.

7. W. Dungchai, O. Chailapakul, and C. S. Henry, Anal. Chim. Acta, 2010, 674, 227.

8. X. Li, J. Tian, T. Nguyen, and W. Shen, Anal. Chem., 2008 , 80, 9131.

9. P. Rattanarat, W. Dungchai, D. Cate, J. Volckens, O. 
Chailapakul, and C. S. Henry, Anal. Chem., 2014, 86, 3555.

10. E. Carrilho, A. W. Martinez, and G. M. Whitesides, Anal. Chem., 2009, 81, 7091.

11. K. Ogawa and T. Kaneta, Anal. Sci., 2016, 32, 31.

12. D. A. Bruzewicz, M. Reches, and G. M. Whitesides, Anal. Chem., 2008, 80, 3387.

13. D. Zang, L. Ge, M. Yan, X. Song, and J. Yu, Chem. Commun. (Camb)., 2012, 48, 4683.

14. S. Wang, L. Ge, X. Song, J. Yu, S. Ge, J. Huang, and F. Zeng, Biosens. Bioelectron., 2012, 31, 212.

15. P. de Tarso Garcia, T. M. Garcia Cardoso, C. D. Garcia, E. Carrilho, and W. K. Tomazelli Coltro, RSC Adv., 2014, 4, 37637.

16. T. M. G. Cardoso, P. T. Garcia, and W. K. T. Coltro, Anal. Methods, 2015, 7, 7311.

17. V. F. Curto, N. Lopez-Ruiz, L. F. Capitan-Vallvey, A. J.
Palma, F. Benito-Lopez, and D. Diamond, RSC Adv., 2013, 3, 18811 .

18. W. Liu, Y. Guo, J. Luo, J. Kou, H. Zheng, B. Li, and Z. Zhang, Spectrochim. Acta, Part A, 2015, 141, 51.

19. E. Fu, T. Liang, P. Spicar-Mihalic, J. Houghtaling, S. Ramachandran, and P. Yager, Anal. Chem., 2012, 84, 4574.

20. E. M. Fenton, M. R. Mascarenas, G. P. López, and S. S. Sibbett, ACS Appl. Mater. Interfaces, 2009, 1, 124.

21. W. Liu, C. L. Cassano, X. Xu, and Z. H. Fan, Anal. Chem., 2013, 85, 10270.

22. W. Liu, J. Luo, Y. Guo, J. Kou, B. Li, and Z. Zhang, Talanta, 2014, 120, 336.

23. L. S. A. Busa, M. Maeki, A. Ishida, H. Tani, and M. Tokeshi, Sens. Actuators, B, 2016, 236, 433.

24. P. D. Josephy, T. Eling, and R. P. Mason, J. Biol. Chem., 1982, 257, 3669 